\title{
Correction to: Optimal Decision Criteria for the Study Design and Sample Size of a Biomarker-Driven Phase III Trial
}

\author{
Akira Takazawa $^{1,2} \cdot$ Satoshi Morita ${ }^{2}$
}

Published online: 14 April 2020

(C) The Drug Information Association, Inc 2020

\section{Correction to: Therapeutic Innovation \& Regulatory Science https://doi.org/10.1007/s43441-020-00119-1}

In the original article in the section "Application to the Motivating Example", Greenberg's trial data have been deleted from the historical data. Tables 3, 4, and 5 along with corresponding text in the manuscript have been updated using Risner's trial data as the single historical data set. Greenberg's trial was a crossover study; the values of $\sigma^{2}$ calculated from this study was the within-subjects variance, not the between-subjects variance. Thus, it was not appropriate to calculate the between-subjects variance and derive the predictive distribution including Greenberg's trial data.

Updated Tables 3, 4, and 5 are below, along with updated manuscript text.

\section{Application to the Motivating Example}

Here, we show what decisions are made when we apply the proposed method to the motivating example described above. Table 3 shows the result of clinical trial that evaluated the change from baseline in ADAS-cog compared to placebo in a randomized study. Note that a negative number indicates the improvement from the observed ADAS-cog at baseline. The estimates of variance of treatment effect in each ApoE4 status were calculated using the sample size in each arm and the result to derive a predictive distribution of treatment effect.

Using the value presented in Table 3, we calculated the standard deviation of response in each arm and each population as $\sigma=4.94$ and we set the between-trial standard deviation as $\tau=0.25$, a small between-trial variance of response $(\sigma / \tau=20)$, and $\tau=0.99$, a large between-trial variance

Table 3. Result of Clinical Trial in the Motivating Example.

\begin{tabular}{lcccccc}
\hline & & \multicolumn{2}{c}{ Sample Size $^{\mathrm{a}}$} & & & \multicolumn{2}{c}{$\begin{array}{c}\text { Estimates of Variance of } \\
\text { Tistorical Data }\end{array}$} & ApoE4 Status & & Arm E & Arm P & & Result & $\begin{array}{c}\text { Treatment Effect } \\
\text { Risner et al. }{ }^{11}\end{array}$ \\
& Positive & 36 & 35 & $1.49[-0.81,3.80]$ & 1.34 \\
& Negative & 42 & 43 & $-2.94[-5.06,-0.81]$ & 1.15 & \\
\hline
\end{tabular}

${ }^{a}$ Arm E and Arm P denote an Experimental Arm and a Placebo Arm, respectively. The Experimental Arms are Rosiglitazone and Donepezil in Risner et al., ${ }^{11}$ respectively.

The original article can be found online at https://doi.org/10.1007/ s43441-020-00119-1.

Akira Takazawa

takazawa@ono.co.jp

1 Data Science Department, ONO Pharmaceutical Co., Ltd, 8-2, Kyutaromachi 1-Chome, Chuo-ku, Osaka 541-8564, Japan

2 Department of Biomedical Statistics and Bioinformatics, Kyoto University Graduate School of Medicine, Kyoto, Japan 
Table 4. Predictive Distribution of Treatment Effects in Each Population.

\begin{tabular}{lll}
\hline Populations & \multicolumn{1}{c}{$\tau=0.25$} & \multicolumn{1}{c}{$\tau=0.99$} \\
\hline ApoE4-positive & $\mathrm{N}(1.49,1.59)$ & $\mathrm{N}(1.49,5.28)$ \\
ApoE4-negative & $\mathrm{N}(-2.94,1.40)$ & $\mathrm{N}(-2.94,5.09)$ \\
Full & $\mathrm{N}(-0.06,0.84)$ & $\mathrm{N}(-0.06,2.85)$ \\
\hline
\end{tabular}

\begin{tabular}{llccc}
\hline$\tau$ & \multicolumn{1}{c}{ Designs } & $\begin{array}{c}\text { Optimal Sample } \\
\text { Size (total) }\end{array}$ & PoS & eNPV \\
\hline 0.25 & Traditional & N/A & N/A & N/A \\
& Enriched & 80 & 0.74 & 1538 \\
& Stratified & 310 & 0.77 & 1658 \\
& Adaptive enrichment & 180 & 0.80 & 1721 \\
0.99 & Traditional & N/A & N/A & N/A \\
& Enriched & 130 & 0.71 & 606 \\
& Stratified & 370 & 0.70 & 1161 \\
& Adaptive enrichment & 190 & 0.70 & 1430 \\
\hline
\end{tabular}

N/A Indicates that the PoS of the design did not exceed 0.70 in the Maximum Sample Size.
Table 5. The Optimal Sample Size, PoS, and eNPV in the Motivating Example.
The eNPV was higher in the order of the adaptive enrich-

$(\sigma / \tau=5)$. The predictive distributions of treatment effects in ApoE4-positive, ApoE4-negative, and full populations are derived by using formula (1) and above values, and presented in Table 4. Note that the prevalence of the ApoE4positive population $\lambda$ and ApoE4-negative population $(1-\lambda)$ are set as 0.65 and 0.35 , respectively. ${ }^{27}$

To calculate the eNPV, we also need to set the parameters related to the study design, the development cost, and the revenue. The values of each parameter used to calculate the eNPV are presented in Table A3 of Appendix 4. Table 5 shows the optimal sample size, PoS, and eNPV in each design. ment design, stratified design, enriched design, and traditional design, regardless of the value of $\tau$. Therefore, the adaptive enrichment design is the optimal design in the motivating example and we decided to use the adaptive enrichment design in the phase III confirmatory study.

Publisher's Note Springer Nature remains neutral with regard to jurisdictional claims in published maps and institutional affiliations. 